\title{
GCDFP-I 5,AR, and Her-2 as biomarkers for primary ductal adenocarcinoma of the lacrimal gland: a Chinese case and literature review
}

This article was published in the following Dove Press journal:

OncoTargets and Therapy

II May 2015

Number of times this article has been viewed

\section{Miao-Miao Zhu' \\ Hong-Guang Cui' \\ Xiao-Dong Teng ${ }^{2}$}

'Department of Ophthalmology, ${ }^{2}$ Department of Pathology, The First Affiliated Hospital, School of Medicine, Zhejiang University, Hangzhou, People's Republic of China
Correspondence: Hong-Guang Cui Department of Ophthalmology, The First Affiliated Hospital, School of Medicine, Zhejiang University, 79 Qingchun Road, Hangzhou 310003, People's Republic of China

Tel $+8657 \mid 87236790$

Fax +86 57| 87236393

Email dr_chg@।63.com
Purpose: Primary ductal adenocarcinoma (PDA) of the lacrimal gland is a rare malignant epithelial tumor, and its clinicopathological characteristics are still unclear. This study aimed to report a novel case of PDA of the lacrimal gland in the People's Republic of China, as well as to determine its histopathological and immunohistochemical characteristics to support early diagnosis and direct further therapy.

Patient and methods: Clinical data (including ocular examination, computed tomography, magnetic resonance imaging, positron emission tomography, mammography, and serum tumor marker examination) and treatment of a 49-year-old woman with a left lacrimal gland mass, which was diagnosed as PDA of the lacrimal gland, were reported. Histopathological and immunohistochemical studies were performed. Eleven papers regarding this uncommon neoplasm were reviewed.

Results: Histopathologically, most of the tumor cells featured abundant granular eosinophilic cytoplasm, while few of them had a frothy appearance. The mass showed breast ductal carcinomalike structural features, which most commonly demonstrated central necrosis, while less of these features showed cord-like infiltration. Immunohistochemically, the tumor cells were positive for GCDFP-15, CK 18 (++), AR (90\%), Her-2 (+++), P53 (100\%), and Ki-67 (with a proliferation index approximately 60\%), while they were negative for ER, PR, P63, calponin, and CD 117.

Conclusion: This was the first Chinese case of PDA of the lacrimal gland that had been reported. We suggested that GCDFP-15, AR, and Her-2 should be tested as biomarkers for ductal adenocarcinoma of lacrimal gland to confirm diagnosis, guide therapy, and further predict prognosis.

Keywords: GCDFP-15, androgen receptors, Her-2, ductal adenocarcinoma, lacrimal gland, orbital tumor

\section{Introduction}

Primary ductal adenocarcinoma (PDA) of the lacrimal gland is a high-grade malignant epithelial tumor that arises in the upper eyelid and generally presents as a mass accompanied by symptoms such as exophthalmos, pseudoptosis, dystopia, and so on. It is extremely rare, as it accounts for only $2 \%$ of epithelial lacrimal gland tumors. ${ }^{1}$ To our knowledge, there are only 14 de novo cases and one case of ductal adenocarcinoma ex pleomorphic adenoma reported in the literature. ${ }^{2-12}$ It is important to diagnose ductal adenocarcinomas of the lacrimal gland in their early stages, as they are highly aggressive. However, the clinicopathological characteristics of ductal adenocarcinoma of lacrimal gland are still unclear. In this study, we aimed to report a novel case of PDA of the lacrimal gland in the People's Republic of China, and we also determined its immunohistochemical characteristics to support its early diagnosis. 


\section{Patient and methods}

\section{Clinical data}

The patient's clinical data were reviewed, including findings from an ocular examination, computed tomography (CT) scan, magnetic resonance imaging (MRI) scan, whole-body positron emission tomography (PET) scan, mammography, serum tumor marker examination, and so on. The seventh edition of the staging manual, prepared by the American Joint Committee on Cancer (AJCC), was used to determine the tumor-necrosis-metastasis category of the patient. ${ }^{13}$ The study was approved by the Human Subjects Ethics Subcommittee of Zhejiang University, and it adhered to the tenets of the Declaration of Helsinki. Written informed consent was obtained from the patient.

\section{Histopathology and immunohistochemistry}

Biopsy and surgical samples were collected and embedded in paraffin for histological and immunohistochemical analyses. Immunohistochemical studies for GCDFP-15 (mouse monoclonal antibody; Dako Denmark A/S, Glostrup, Denmark), CK 18 (mouse monoclonal antibody; Abcam plc, Cambridge, UK), AR (mouse monoclonal antibody; BioGenex, San Ramon, CA, USA), Her-2 (polyclonal antibody; Dako Denmark A/S), p53 (Novocastra; Leica Mycrosystems GmbH, Wetzlar, Germany), Ki-67 (Dako Denmark A/S), ER (mouse monoclonal antibody; BioGenex), PR (mouse monoclonal antibody; BioGenex), P63 (mouse monoclonal antibody; Abcam plc), calponin (rabbit monoclonal antibody; Abcam plc), and CD 117 (rabbit polyclonal; Dako Denmark A/S) were performed on the paraffin sections.

\section{Literature review}

We searched PubMed for published studies that reported on "ductal adenocarcinoma" or "duct carcinomas" of the lacrimal gland. Eleven relevant papers were found. The clinical data including age, sex, country, treatment, immunohistochemical results, and follow-ups were summarized.

\section{Results}

\section{Case report}

A 49-year-old woman presented in June 2014 with gradual progression of exophthalmos of the left eye, a painless palpable mass under the left orbital rim, double vision, and epiphora for the last 6 months. The patient's visual acuity was 20/20 in the right eye and 20/25 in the left eye. Hertel exophthalmometry was $12 \mathrm{~mm}$ for the right eye and $15 \mathrm{~mm}$ for the left eye. The mass was palpated at the superior, temporal, and inferior orbital rim of the left eye featuring well-defined and regular margins, a smooth surface, and it was slightly hard. Movement of the left eye was restricted in the left field of gaze. Examination of the globe did not show obvious abnormality. The intraocular pressure was $13 \mathrm{mmHg}$ in the right eye and $15 \mathrm{mmHg}$ in the left eye.

CT scans showed an irregular orbital mass with ill-defined margins, and featured low and heterogeneous density in the extraconal space of the left orbit (Figure 1). Figure 1A, 1B and $1 \mathrm{C}$ are different $\mathrm{CT}$ sections to show the shape of mass. The lesion contained an ill-circumscribed calcification with a size of $11 \times 7 \mathrm{~mm}$ near the lateral rectus muscle (Figure 1B, C). The upper eyelid was involved and the surrounding soft tissue was thickened. The medial wall of the left orbit showed local destruction (Figure 1D). On MRI, the mass appeared hypointense on T1WI and T2WI, which was enhanced following contrast administration (Figure 2). Serum tumor marker examination showed increased CEA $(8.4 \mathrm{ng} / \mathrm{mL})$ expression.

The patient was diagnosed with an orbital carcinoma (nature undefined) in the left orbit. She then underwent excisional biopsy. A histopathological examination revealed a poorly differentiated adenocarcinoma.

Whole-body PET, which was performed to determine the degree of metastasis, showed irregular and slightly elevated fluorodeoxyglucose uptake by the left orbital mass with a maximum standardized uptake value of approximately 3.1. The lesion grew in the extraconal space, involving the superior rectus, lateral rectus, inferior oblique, and internal rectus muscles. No systemic metastasis and bone destruction were present in this case (Figure 3). Mammography examination showed hyperplasia of the mammary glands (figure not shown).

Exenteration with radical orbitectomy and postoperative radiotherapy was performed 1 month after biopsy. The specimens underwent histopathological and immunohistochemical analyses. The patient had no evidence of recurrence or metastasis during the 9-month follow-up.

\section{Histopathology and immunohistochemistry}

Histopathologically, most of the tumor cells featured abundant granular eosinophilic cytoplasm, while few of them had a frothy appearance. The mass showed breast ductal carcinoma-like structural features, which most commonly demonstrated central necrosis; only a few of its cells exhibited cord-like infiltration (Figure 4). The mass originated in the lacrimal gland and involved periocular adipose tissue, striated muscle, and nervous tissue. Immunohistochemically, 

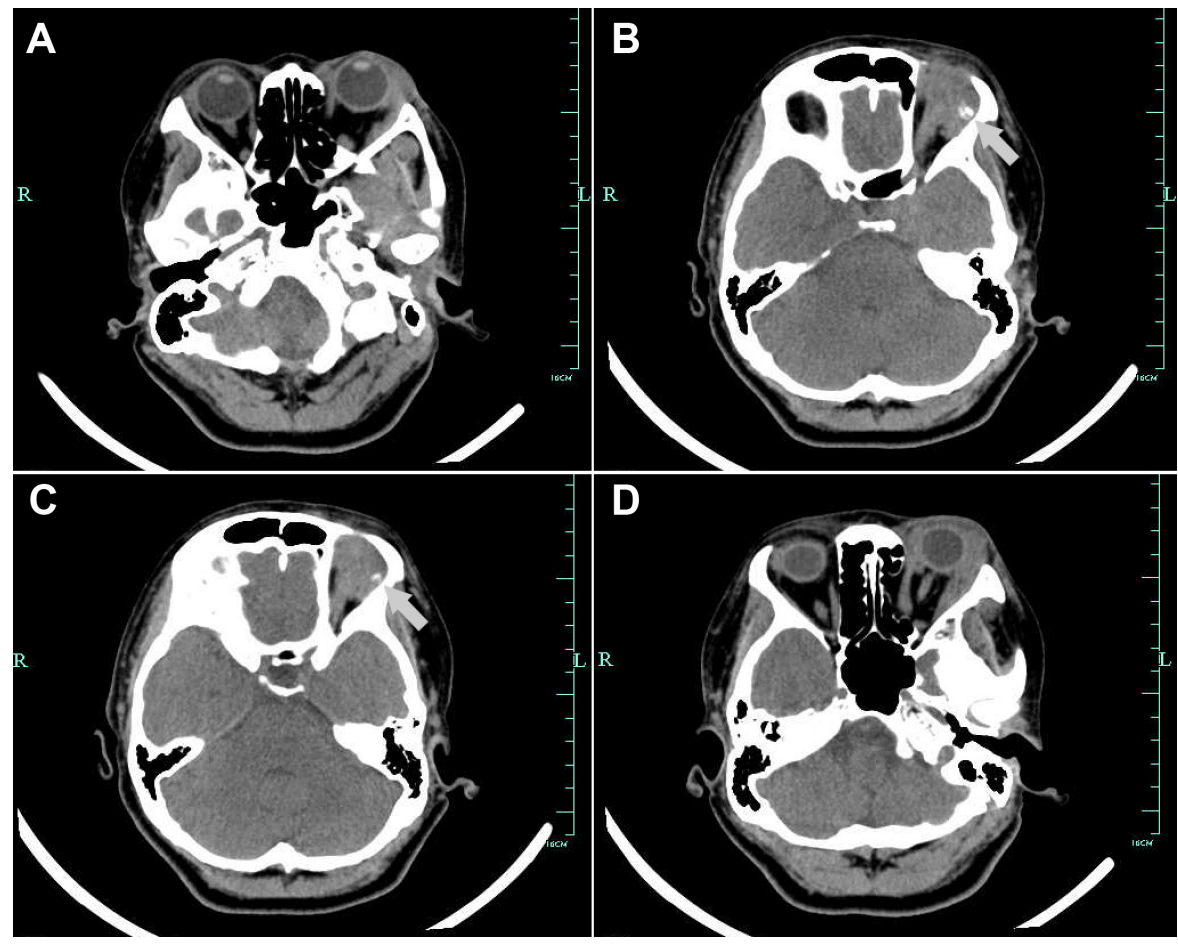

Figure I Computed tomography (CT) scan shows an irregular orbital mass with ill-defined margins, as well as low and heterogeneous density in the extraconal space of the left orbit.

Notes: (A-C) Represent different CT sections to show the shape of the mass. The lesion contains an ill-circumscribed calcification with a size of II $\times 7 \mathrm{~mm}$ near the lateral rectus muscle (arrow; B, C). The medial wall of the left orbit shows local destruction (D).

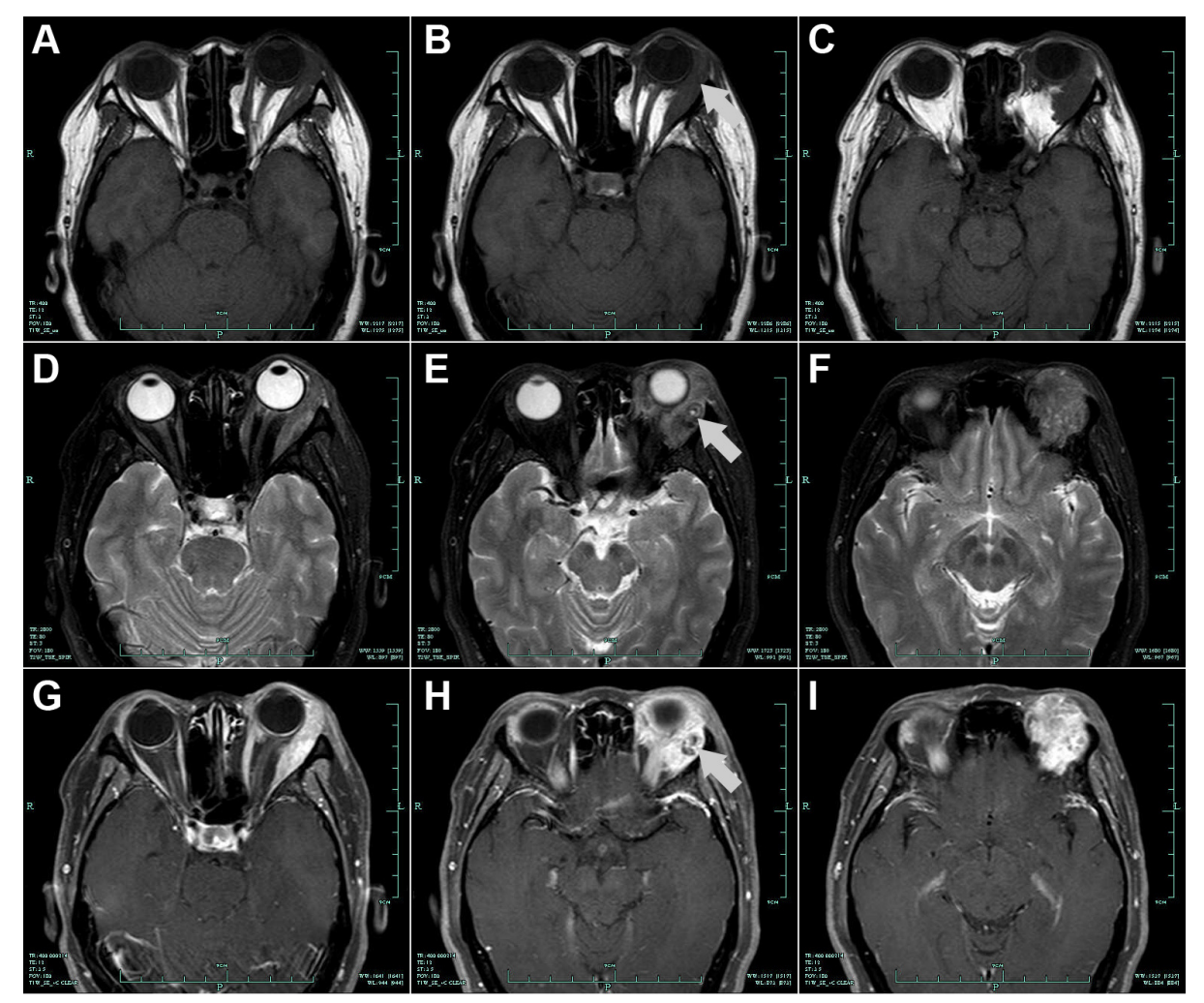

Figure 2 Magnetic resonance imaging scans showed an irregular orbital mass with ill-defined margins in the extraconal space of the left orbit.

Notes: The mass appeared hypointense on TIWI (A-C) and T2WI (D-F), which was enhanced following contrast administration (G-I). An ill-circumscribed calcification is shown near the lateral rectus muscle (B, E, H; arrow). 


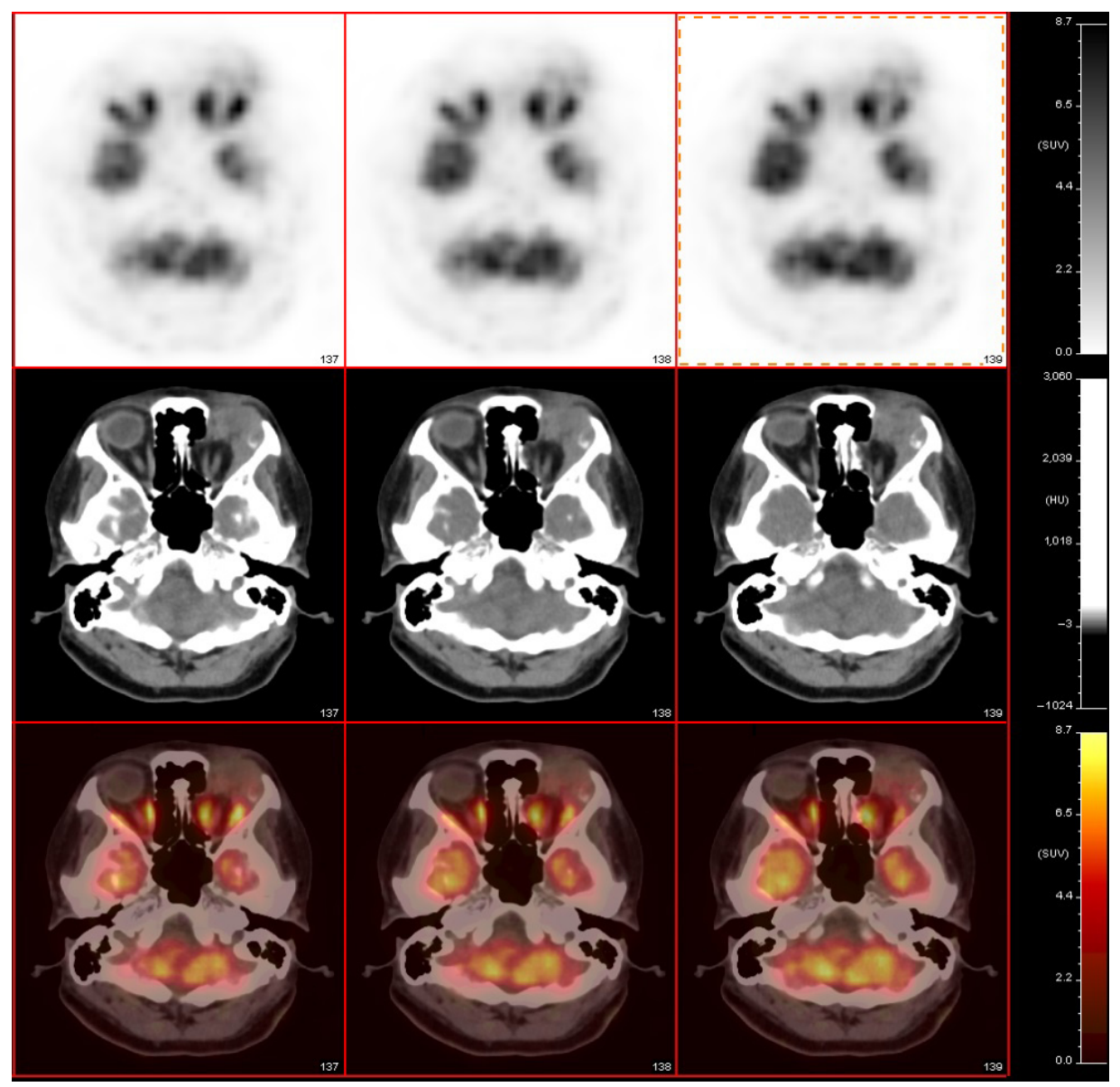

Figure 3 Whole-body positron emission tomography shows irregular and slightly elevated fluorodeoxyglucose uptake by the left orbital mass with a maximum standardized uptake value approximately 3.1 .

Note: The lesion had grown in the extraconal space, involving the superior rectus, lateral rectus, inferior oblique, and internal rectus muscles.

Abbreviations: SUV, standardized uptake value; $\mathrm{HU}$, hounsfield unit.

the tumor cells were positive for GCDFP-15/BRST-2, CK 18 (++), AR (90\%), Her-2 (+++), P53 (100\%), and Ki-67 (with a proliferation index approximately $60 \%$ ) (Figure 5), while they were negative for ER, PR, P63, calponin, and CD 117 (data not shown). The results of the analyses are summarized in Table 1. According to the histopathological and immunohistochemical findings, the mass was diagnosed as poorly differentiated ductal adenocarcinoma of the lacrimal gland, T1cN0M0 according to the 2010 AJCC staging system.

\section{Discussion}

Ductal adenocarcinoma of the lacrimal gland was first reported by Katz et al in $1996 .{ }^{12}$ Only 15 cases have been reported in the literature in the past 18 years. Since the number of cases is sparse, the characteristics of ductal adenocarcinoma of lacrimal gland are still unclear, which render diagnosis difficult, and misdiagnosis is increased. In this study, we reported a new case of a patient suffering from ductal adenocarcinoma of the lacrimal gland. This was the first case reported in a Chinese patient. Our immunohistochemical analysis discovered that lacrimal ductal adenocarcinoma was positive for GCDFP-15, AR, and Her-2. It also indicated that GCDFP-15, AR, and Her-2 were biomarkers for ductal adenocarcinoma of lacrimal gland.

With respect to orbital diseases, inflammation, lymphoid proliferations, and lacrimal gland tumors can all present with exophthalmos, a palpable orbital mass, double vision, or epiphora. CT/MRI could help refine the differential diagnosis. In the present study, an ill-circumscribed calcification within the mass was shown upon CT imaging (Figure 1B and C). It had been reported that benign pleomorphic adenomas and malignant mixed ex pleomorphic adenomas showed atypical calcification upon imaging. ${ }^{14}$ Although it had not been previously confirmed, we speculated that calcification could be an atypical imaging feature of ductal adenocarcinoma of lacrimal gland. In order to confirm whether bone destruction was a feature of ductal adenocarcinoma of lacrimal gland, as 

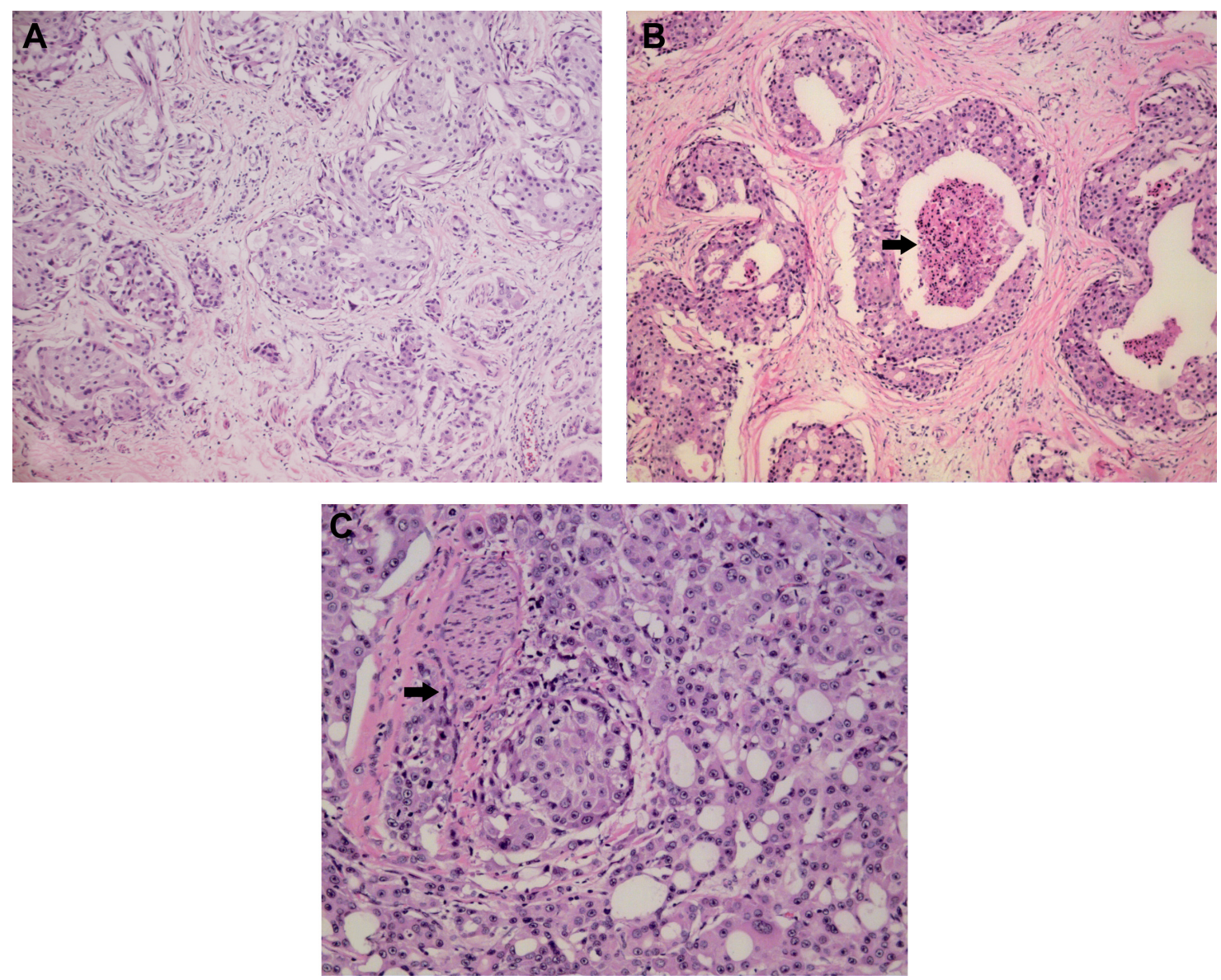

Figure 4 Histopathological sections of the mass.

Notes: (A) The mass shows breast ductal carcinoma-like structural features. The tumor cells have abundant granular eosinophilic cytoplasm, while a few of them have a frothy appearance (hematoxylin and eosin staining; original magnification: I00x); (B) intraductal necrosis is demonstrated in the mass (arrow; hematoxylin and eosin staining; original magnification: 100x); (C) nervous tissue is involved (arrow; hematoxylin and eosin staining; original magnification: 200x).

shown on CT imaging (Figure 1D), we tested the tissue near the local destruction of the orbital wall. Both histopathological and immunohistochemical tests were negative (data not shown). This indicated that local destruction of the medial wall of the left orbit was not caused by invasion of the tumor; it might be because of compression from the tumor.

When compared with CT/MRI imaging, the histopathological and immunohistochemical analyses were the most valuable techniques for diagnosing ductal adenocarcinoma of the lacrimal gland. In the present study, the mass showed breast ductal carcinoma-like structural features with central necrosis, and the tumor cells had abundant granular eosinophilic cytoplasm, while few of them had a frothy appearance (Figure 4). These histopathological characteristics were similar to those of cases reported previously., ${ }^{2,3}$ To further confirm the diagnosis, eleven antigens were tested immunohistochemically, of which GCDFP-15, CK 18, AR, Her-2, P53, and Ki-67 were positive (Figure 5), while ER, PR, P63, calponin, and CD 117 were negative (data not shown). The results were consistent with those of previous studies (Table 1). ${ }^{3,4,6,9}$ To confirm the origin of the tumor, PET and mammography examination were performed. PET showed that only the left orbital mass showed a high level of uptake of fluorodeoxyglucose; the other organs did not. Mammography showed hyperplasia of the mammary glands, but there was no evidence of tumor. The increased serum CEA $(8.4 \mathrm{ng} / \mathrm{mL})$ returned to a normal level $(3.3 \mathrm{ng} / \mathrm{mL})$ after the patient submitted to exenteration with radical orbitectomy. This confirmed that the ductal adenocarcinoma had originated from the lacrimal gland but not from the breast or other organs.

As is currently known, PDA of lacrimal gland is the counterpart of salivary duct carcinoma, resembling an invasive ductal carcinoma of the breast. ${ }^{2}$ As there were few studies published on PDA, we borrowed concepts from the histopathologically similar invasive ductal carcinoma or salivary duct carcinoma, which were well studied. In this report, GCDFP-15 was tested in lacrimal ductal adenocarcinoma for the second time. It was first reported in Milman et al's 

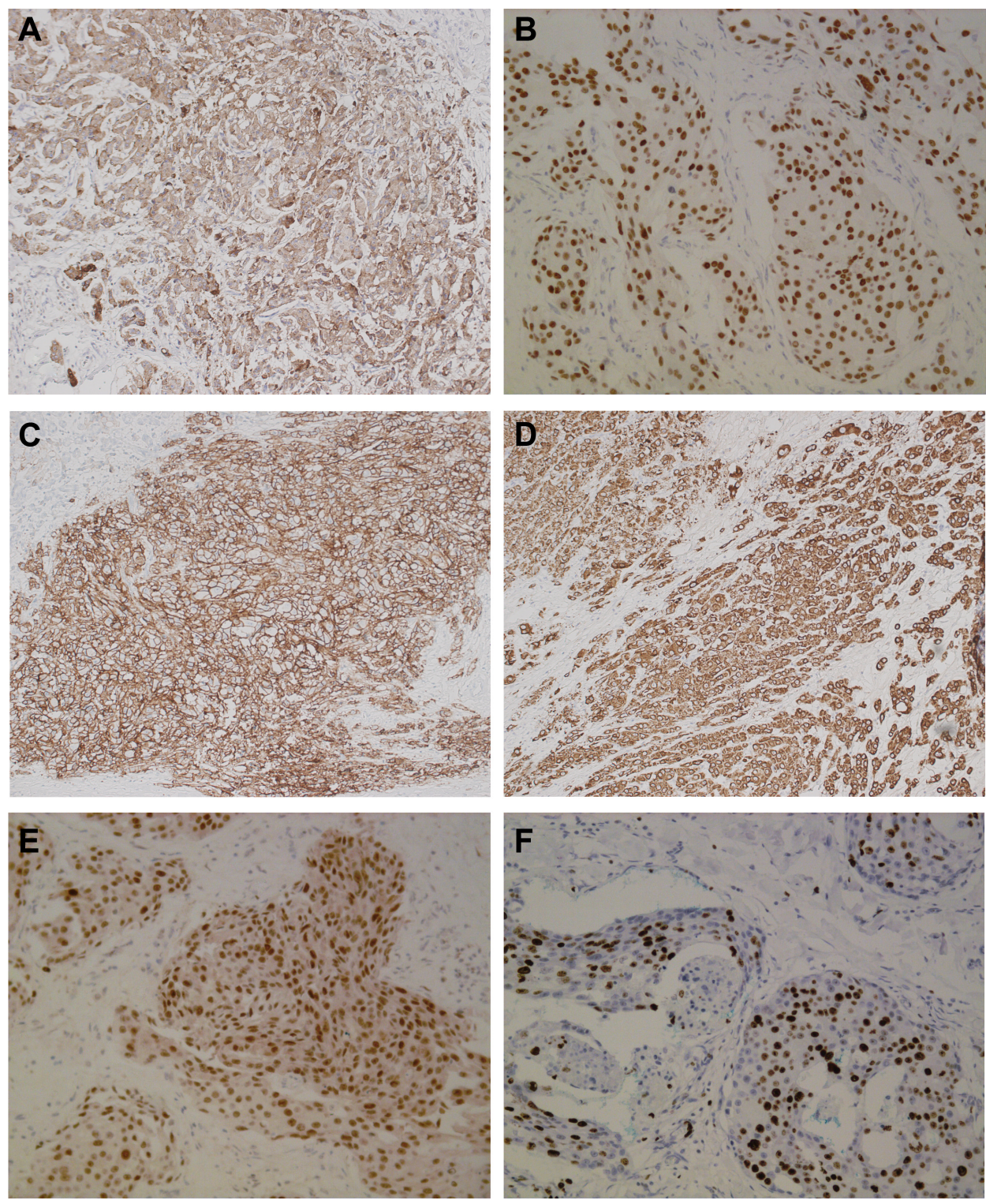

Figure 5 Immunohistochemical staining of the mass.

Notes: The tumor cells were positive for (A) GCDFP-I5; (original magnification: I00x); (B) AR; 90\%; (original magnification: 200×); (C) Her-2 (+H); (original magnification: 100x); (D) CK 18 (++); (original magnification: I00x); (E) P53 (100\%; original magnification: 200x); and (F) Ki-67 (with a proliferation index approximately 60\%; original magnification: 200×). The tumor cells were negative for ER, PR, P63, calponin, and CD 117 (data not shown).

study, ${ }^{8}$ which concerned lacrimal ductal adenocarcinoma and was published in 2005. GCDFP-15 was reported as a characteristic biomarker for mammary differentiation during histopathology, which was also expressed in serous cells of the submandibular salivary gland, and accessory lacrimal glands. ${ }^{15}$ Research has shown that the expression of GCDFP-15 was consistent with pro-apoptotic and antiproliferative genes. ${ }^{16}$ According to Darb-Esfahani et al's study, GCDFP-15 was regulated by the AR and was linked to Her-2 in breast carcinomas. The authors suggested that GCDFP-15 was a favorable prognostic factor in breast carcinomas, but the impact was not independent from other factors such as AR and Her-2. ${ }^{17}$ According to Kubota et al's study, ${ }^{3}$ AR was suggested as a diagnostic marker for ductal adenocarcinoma of lacrimal gland because all of the samples obtained from patients were AR-positive. Both AR and Her-2 were positive in the present immunohistochemical study. In all the samples, we suggested that GCDFP-15, AR, and Her-2 should be tested in the PDA of the lacrimal gland to confirm diagnosis and further predict prognosis. In addition, more research is needed to confirm the predictive effect of these markers.

We reviewed the literature that had been published about PDAs of the lacrimal gland. Including the present case, 


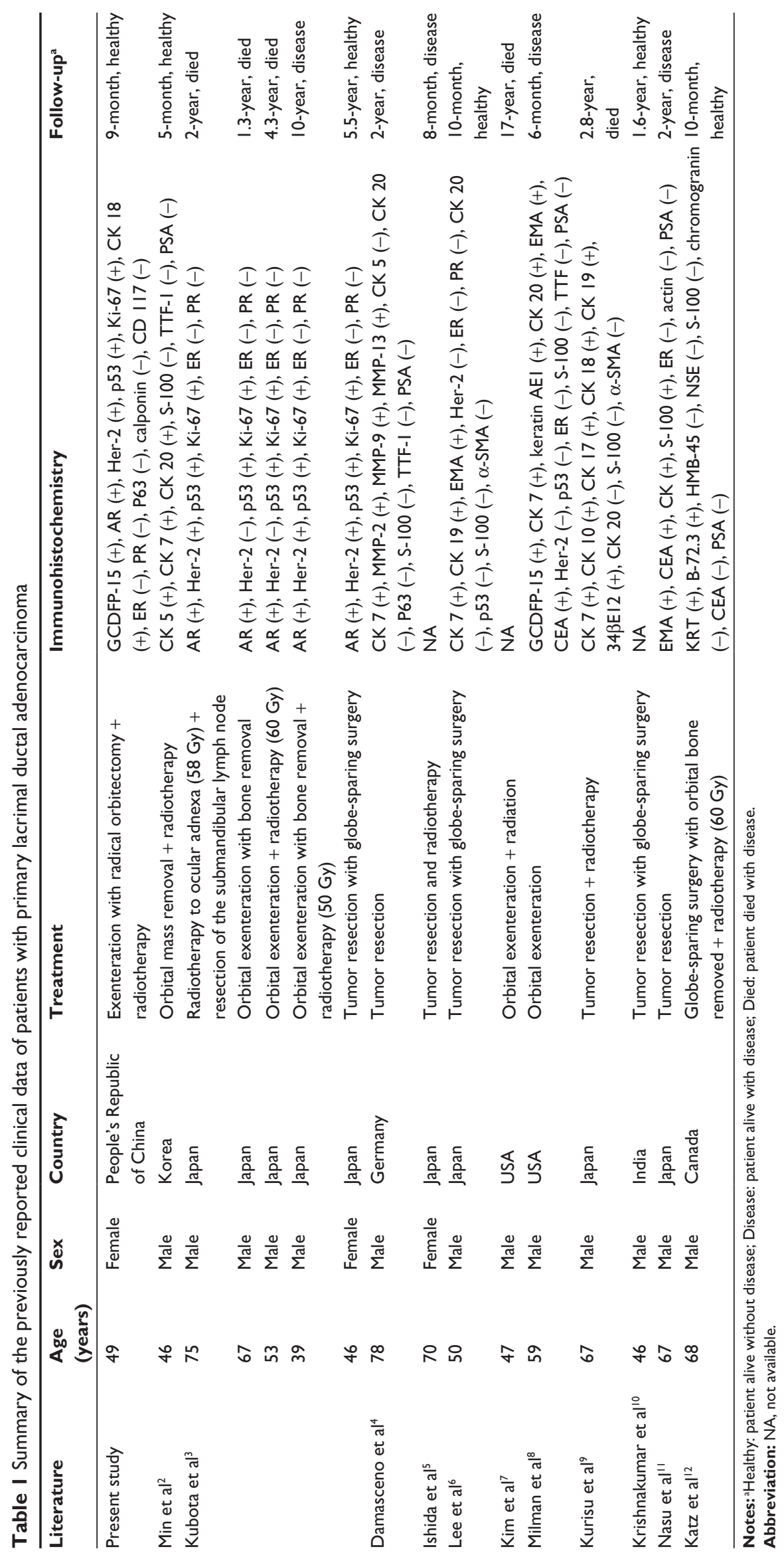


there were a total of 15 de novo cases and one case of ductal adenocarcinoma ex pleomorphic adenoma. The clinical data including age, sex, country, treatment, immunohistochemistry, and follow-ups are summarized in Table 1. Among these cases, 13 patients were male while three were female. It has been suggested that ductal adenocarcinoma of the lacrimal gland predominantly affects men, with a male-to-female ratio of $4.3: 1$. Furthermore, the median age of patients was 56 years with a range from 39 to 78 years. Among 16 cases, nine were from Japan, two were from the USA, and the remaining patients were from other countries (one case each in the People's Republic of China, India, Korea, Germany, and Canada). It was indicated that ductal adenocarcinoma of the lacrimal gland appeared primarily in Asia, especially in Japan. The treatment that had been reported for lacrimal ductal adenocarcinoma included surgical operations with or without postoperative radiotherapy. It was difficult to confirm whether the combination therapy would increase the 5-year survival rate of patients because the tumor stages and follow-up durations had varied among the different studies.

\section{Conclusion}

In conclusion, this study represents the first case of a ductal carcinoma of the lacrimal gland reported in the People's Republic of China. We suggest that GCDFP-15, AR, and Her-2 be tested as biomarkers of ductal adenocarcinoma of lacrimal gland to confirm diagnosis, direct further therapy, and predict prognosis. Further studies are needed to clarify the relationship between immunohistochemistry, therapy, and patient prognosis.

\section{Acknowledgments}

The work was supported by a grant from Natural Science Foundation of Zhejiang Province (LY12H12009). The funding organization had no role in the design or conduct of this research. English-language editing of this manuscript was provided by Journal Prep.

\section{Disclosure}

The authors declare that they have no conflicts of interest in this work.

OncoTargets and Therapy

\section{Publish your work in this journal}

OncoTargets and Therapy is an international, peer-reviewed, open access journal focusing on the pathological basis of all cancers, potential targets for therapy and treatment protocols employed to improve the management of cancer patients. The journal also focuses on the impact of management programs and new therapeutic agents and protocols on

\section{References}

1. Weis E, Rootman J, Joly TJ, et al. Epithelial lacrimal gland tumors: pathologic classification and current understanding. Arch Ophthalmol. 2009;127(8):1016-1028.

2. Min KW, Park HK, Kim WY, et al. Primary Ductal Adenocarcinoma of the Lacrimal Gland, associated with Abundant Intracytoplasmic Lumens containing Some Eosinophilic Hyaline Globules: Cytological, Histological and Ultrastructural Findings. Ultrastruct Pathol. 2014;38(5):363-366.

3. Kubota T, Moritani S, Ichihara S. Clinicopathologic and immunohistochemical features of primary ductal adenocarcinoma of lacrimal gland: five new cases and review of literature. Graefes Arch Clin Exp Ophthalmol. 2013;251(8):2071-2076.

4. Damasceno RW, Holbach LM. Primary ductal adenocarcinoma of the lacrimal gland: case report. Arq Bras Oftalmol. 2012;75(1):64-66.

5. Ishida M, Hotta M, Kushima R, et al. Case of ductal adenocarcinoma ex pleomorphic adenoma of the lacrimal gland. Rinsho Byori. 2009; 57(8):746-751.

6. Lee YJ, Oh YH. Primary ductal adenocarcinoma of the lacrimal gland. Jpn J Ophthalmol. 2009;53(3):268-270.

7. Kim MJ, Hanmantgad S, Holodny AI. Novel management and unique metastatic pattern of primary ductal adenocarcinoma of the lacrimal gland. Clin Experiment Ophthalmol. 2008;36(2):194-196.

8. Milman T, Shields JA, Husson M, et al. Primary ductal adenocarcinoma of the lacrimal gland. Ophthalmology. 2005;112(11):2048-2051.

9. Kurisu Y, Shibayama Y, Tsuji M, et al. A case of primary ductal adenocarcinoma of the lacrimal gland: histopathological and immunohistochemical study. Pathol Res Pract. 2005;201(1):49-53.

10. Krishnakumar S, Subramanian N, Mahesh L, Mohan ER, Biswas J. Primary ductal adenocarcinoma of the lacrimal gland in a patient with neurofibromatosis. Eye (Lond). 2003;17(7):843-845.

11. Nasu M, Haisa T, Kondo T, Matsubara O. Primary ductal adenocarcinoma of the lacrimal gland. Pathol Int. 1998;48(12):981-984.

12. Katz SE, Rootman J, Dolman PJ, et al. Primary ductal adenocarcinoma of the lacrimal gland. Ophthalmology. 1996;103(1):157-162.

13. Edge SB, Compton CC. The American Joint Committee on Cancer: the 7th edition of the AJCC cancer staging manual and the future of TNM. Ann Surg Oncol. 2010;17(6):1471-1474.

14. Chawla B, Kashyap S, Sen S, et al. Clinicopathologic review of epithelial tumors of the lacrimal gland. Ophthal Plast Reconstr Surg. 2013;29(6):440-445.

15. Mazoujian G, Pinkus GS, Davis S, et al. Immunohistochemistry of a gross cystic disease fluid protein (GCDFP-15) of the breast. A marker of apocrine epithelium and breast carcinomas with apocrine features. Am J Pathol. 1983;110(2):105-112.

16. Debily MA, Marhomy SE, Boulanger V, et al. A functional and regulatory network associated with PIP expression in human breast cancer. PLoS One. 2009;4(3):e4696.

17. Darb-Esfahani S, von Minckwitz G, Denkert C, et al. Gross cystic disease fluid protein 15 (GCDFP-15) expression in breast cancer subtypes. BMC Cancer. 2014;14:546.

\section{Dovepress}

patient perspectives such as quality of life, adherence and satisfaction. The manuscript management system is completely online and includes a very quick and fair peer-review system, which is all easy to use. Visit http://www.dovepress.com/testimonials.php to read real quotes from published authors. 